\title{
High Energy behaviour of Neutron Star Low Mass $X$-ray binary systems seen by INTEGRAL
}

\section{Antonella Tarana, ${ }^{* \dagger}$ Angela Bazzano, Pietro Ubertini and on behalf of the IBIS Survey team}

IASF-Rome/INAF, via Fosso del cavaliere 100, 00133 Rome, Italy

E-mail: antonella.tarana@iasf-roma.inaf.it

We report on the study of the high energy emission of Neutron Stars in Low Mass X-ray Binary systems performed with INTEGRAL. The X and Gamma behaviour of a selected sample of atoll sources has been monitored through the study of light curves in different energy bands, the construction of hardness-intesity diagrams, and extraction of spectra to discern the physical parameters dominating the spectral variations. We re-constructed the "Bursters Box" with the source analysed plus other persistent bursters and Z sources as revealed by the deep IBIS survey. This allows to compare amongst themselves the sources sample.

The Extreme sky: Sampling the Universe above $10 \mathrm{keV}$ - extremesky2009,

October 13-17, 2009

Otranto (Lecce) Italy

\footnotetext{
* Speaker.

${ }^{\dagger}$ This research has made use of data obtained with INTEGRAL an international collaboration. We acknoweledge the Italian Space Agency financial support via grant ASI-INAF I/008/07 and I/008/06.
} 


\section{Introduction}

This work is the update of the Luminosity diagram presented by the authors in [1] with an increasing number of the Neutron Stars (NSs).

The Luminosity diagram was initially proposed by Barret et al. 1996, 2000, as one of the method (a "luminosity criterion") to distinguish Black Hole (BH) versus NS binaries. The authors have measured the 1-20 keV and 20-200 keV luminosity of a sample of NS and BH systems (optically proved), showing that the X-ray Bursters are clustered in the X-ray Bursters Box at low luminosities ranges with respect to the $\mathrm{BH}$ systems [2] [3]. Other proposed criteria were based on the differences in the X-ray spectra (high energy emission and high temperature of the electrons corona were suggested as indication of a BH nature for the compact object [4] [5]). The observations of NS Low Mass X-ray Binaries (LMXBs) in the hard X-ray band firstly with BeppoSAX and RXTE satellites and now with INTEGRAL, reject these criteria. In fact the observations show that the X-ray spectral parameters and shape, are similar between NSs and BH Candidates. Moreover the detection of hard X-ray tails and high energy emission from the NS systems also (as example in GX 17+2, GX 5+1 [6] [7]), shows that the "luminosity criterion" is difficult to be applied.

The present work confirms that the NSs are high energy emitters with spectra extending well above $100 \mathrm{keV}$, and their spectral states (especially the Hard state) are similar to the BHs ones.

\section{The bursters sample}

We populate the Luminosity Diagram (see section 5) with a selected sample of NS LMXBs analysed in detail with INTEGRAL during last years plus a sample of bursters with persistent behaviour from the IBIS survey [8] and three bursters belonging to the $\mathrm{Z}$ type. The sample analysed and studied in detail individually is composed by bursters and atoll with different characteristics, most of them are located in Globular Cluster, some are transients and some are persistents: $4 \mathrm{U}$ 1608-522, 4U 1728-34, 4U 1722-30, 4U 1820-30, 4U 1812-12, XB 1832-330 and also $4 U$ 1254-690. Instead the sources with information from the IBIS survey are: 4U 1746-37, XTE J1701-281, 4U 1705-32, IGR 17254-3257, 4U 1246-58, GX 17+2, 4U 1708-40, Cyg X-2, GX 3+1, SLX 1735-269, SLX 1744-299, GX 13+1, Ser X-1, Cir X-1, 4U 1916-053, 4U 0918-54, SLX 1737-282, EXO 0748-676, 4U 1323+62 and 4U 1850-087.

\section{Behaviour of the sources}

The sources 4U 1812-12, XB 1832-330 [9] and 4U 1254-690 [10] showed a persistent flux with a low level of variability $(<25 \%)$ and without any spectral change. In particular, 4U 1812-12 has been detected only in the Hard state up to now [11].

On the contrary we have detected spectral changes from the sources 4U 1820-30 [12], 4U 1608522 [13], 4U 1728-34 [14] and 4U 1722-30 [15]. These changes are associated to transient phenomena for two of them, with spectral hardening and softening occurring in a time scale of daysmonth; the other ones showed continuum emission with high flux variations coupled to spectral transitions. In particular for the sources $4 \mathrm{U} 1820-30$ and 4U 1728-34 an high energy emission characterized by the presence of a hard tail extended above $50 \mathrm{keV}$ was detected for the first time during the hard state [12], [14]. 


\section{Sample observed spectral states}

The performed spectral analysis can be summarized with the existence of three types of spectral states for the Atoll sources: Soft, Hard and Intermediate.

\subsection{Soft state}

This state (also called "banana" state) is characterised by a strong black body component with temperature of 0.4-0.6 keV plus a Comptonized component with a low electron temperature, $k T_{\mathrm{e}}$, ranging from 2 to $5 \mathrm{keV}$ and high optical depth of 2-9. The input photons of the Comptonization have a temperature of $0.2-1.2 \mathrm{keV}$.

The Soft state of transient sources is characterized by a stronger soft component compared to that of the other persistent sources (as example 4U 1608-522 compared to 4U 1254-690). This could be explained by the high flux level reached during the outburst (high luminosity) that implies higher accretion rate and, in turn, high luminosity.

\subsection{Hard state}

The soft black body component during the Hard state (also known as "island" state) is of lower intensity and in some cases is not even detected. The Comptonizing corona shows a higher electron temperature (6-60 keV) and a lower value of the optical depth (0.4-3) than the soft state ones.

The main results of our work is that the Hard states show two different type of behaviour:

In the first one, the corona temperature is high and the spectra extends up to $200 \mathrm{keV}$, and in some cases without a clear high energy cut-off (as for 4U 1608-522 and 4U 1722-30).

In the second one, the corona has a lower temperature and the Comptonization component contributes to the spectrum up to 50-60 keV, while at higher energy $(>50 \mathrm{keV})$ a hard tail, modelled by power law with photon index of 2-2.3, dominates (as for 4U 1820-30 and 4U 1728-34). Alternatively, this state is modeled by hybrid thermal-nonthermal Comptonization (with $k T_{\mathrm{e}}$ of 20$30 \mathrm{keV}$ ). This hard tail could be produced by a synchrotron emission correlated with radio emission from a the base of a jet [16] or more probably from an electron population with hybrid thermal and not thermal velocity distribution [17]. Recently the bluk motion Comptinization applied also in NS [18] represents an alternative physical explanation of the detected hard tails [20].

\subsection{Intermediate state}

These is the state in which sources spend a short time just in coincidence with a spectral state transition. Detection of these states are possible only by a detailed spectral and temporal analysis.

The spectral parameters have intermediate values between the Soft and Hard ones. Nevertheless, it is possible to understand when it is Soft-like or Hard-like by the comparison with the values of the parameters for the two main states.

\section{Luminosity diagram}

We derived the flux values from the best fit models of each source state detected in two energy bands: the soft, 4-10 keV, and the hard, 20-200 keV. We estimated the luminosity in these two 
energy bands using the distances reported in the literature, giving whenever possible priority to the values derived from burst with photospheric radius expansion.

Figure 1 shows the luminosity diagram in the two energy bands for all the sources showing spectral states variation and for sources with persistent behaviour for which average spectrum flux are calculated. We include also the Z sources GX 17+2, Gyg X-2 and GX 13+1 using average spectra even if they are variable source.

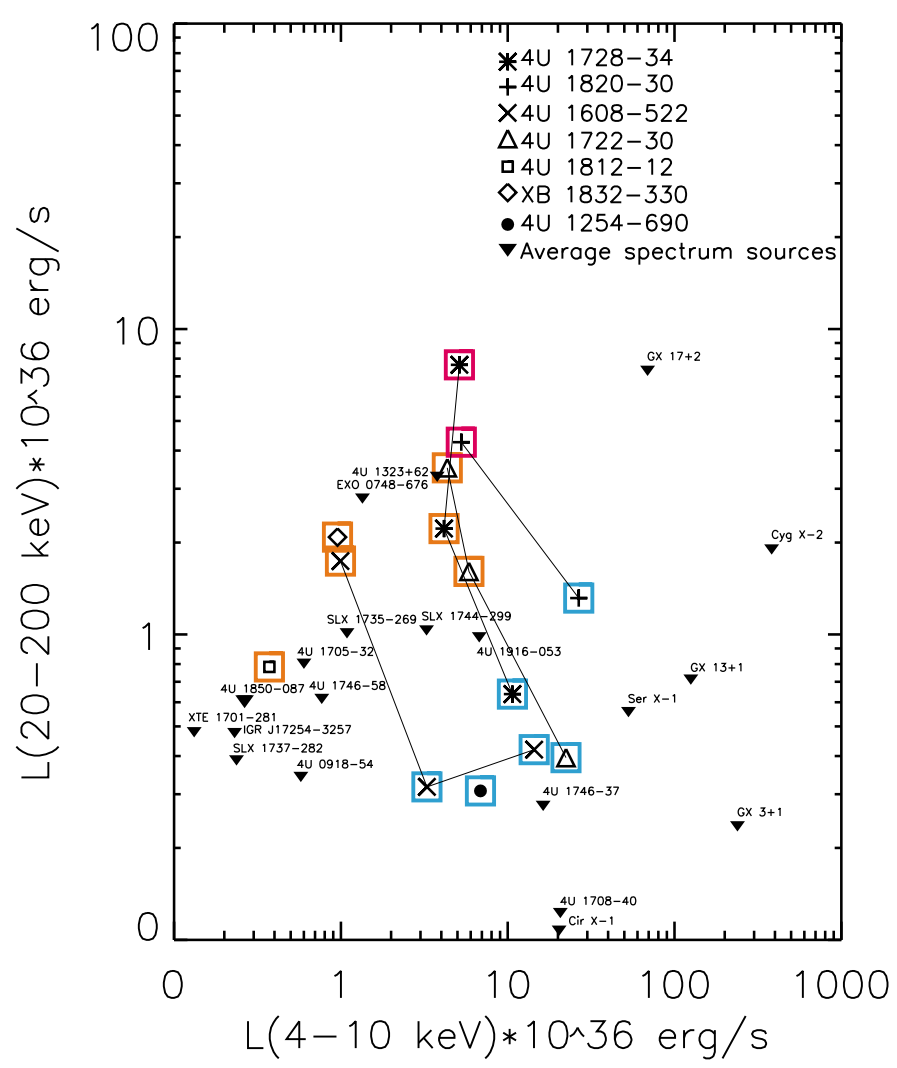

Figure 1: Luminosity diagram of the analysed source sample. For each source the detecte spectral state are shown, joined with a line for the same source. The blue boxes show the Soft states, the orange one the Hard states and the red boxes the Hard states with hard power law tail. The Intermediate states belong to the nearing spectral state-group.

Comparing the luminosity among themselves the different spectral states show the position in different zones. The Soft states indicated by blue boxes are all localized at $\mathrm{L}_{\text {soft }}>3 \times 10^{36} \mathrm{erg} \mathrm{s}^{-1}$ and $\mathrm{L}_{\text {hard }}<1.4 \times 10^{36} \mathrm{erg} \mathrm{s}^{-1}$. On the contrary the Hard states indicated by orange and red boxes are all located at $\mathrm{L}_{\text {soft }}<6 \times 10^{36} \mathrm{erg} \mathrm{s}^{-1}$ and $\mathrm{L}_{\text {hard }}>0.7 \times 10^{36} \mathrm{erg} \mathrm{s}^{-1}$. The Intermediate states observed are located between the Soft and Hard states. By the closeness to the Soft state group or the Hard group we could say that the Intermediate state is associated more to a group than to the other one. As examples, the Intermediate state of $4 \mathrm{U} 1722-30$ and $4 \mathrm{U} 1728-34$ is more similar to the Hard state, while the Intermediate state of 4U 1608-522 to the Soft state group.

The Luminosity diagram seems to be a good clue to characterize the Soft or Hard nature of the 
source spectral states.

We indicate with red boxes the power law - Hard states. They reach high value of $\mathrm{L}_{\text {soft }}, \simeq$ $5 \times 10^{36} \mathrm{erg} \mathrm{s}^{-1}$ and to the highest value of $\mathrm{L}_{\text {hard }},>4 \times 10^{36} \mathrm{erg} \mathrm{s}^{-1}$. Note that the peculiar position of GX 17+2 also (at high value of $\mathrm{L}_{\text {soft }}$ and $\mathrm{L}_{\text {hard }}$ ) is due to the presence of a high energy tail in the evarage spectrum, being this source one of the $\mathrm{Z}$ source already known to show this behaviour as reported by [19],[20].

We investigated the existence of a different luminosity correlation between Soft and Hard states using the Luminosity ratio $R=L_{\text {soft }} / L_{\text {hard }}$. $R$ values ranges from 7.2 to 56.5 for the Soft states, while for the Hard states $R$ values are in the range to 0.45 to 3.64 .

With respect to the Burster Box reported by Barret et al. 2000 [3], our diagram shows in addition the information of the spectral changes, being the luminosities of variable sources not an averaged value but computed for the different spectral states detected. The sources in our sample confirm the existence of a critical value for $\mathrm{L}_{\text {hard }}$ below which the NS systems are positioned in the Bursters Box as reported by Barret et al. $2000\left(L_{c r i t} 1.5 \times 10^{37} \mathrm{erg} \mathrm{s}^{-1}\right)$.

\section{Physical interpretation}

From our analysis it is clear that there is a strong variation in the spectral shape between the hard and soft states of the Atoll sources, probably due to changes in the accretion flow geometry and coupled with variations in the mass accretion rate.

In spite of the difficulty to precisely determine the soft parameters because of the used limited instrumentation band-width (often available only for $\mathrm{E}>4 \mathrm{keV}$ ), we can still draw some remarks and explanations.

The observed "soft component" is modelled either by the diskbb or by bbody emission, and it is not possible to discern the origin of this component that could originate either in the optically thick and geometrically thin accretion disk or NS surface, or optically thick boundary layer between the disk and the NS (i.e. western or eastern model).

The input seed photons necessary for the Comptonization process could in general have the same origin of the soft photons. This hypothesis is not confirmed by our analysis since we have never observed the same temperature value, suggesting a different origin for the seed photons.

During the Hard state the observed disk black body emission is either not detected or has a low temperature value compared to that of the Soft state. This implies a larger extension of the inner radius of the accretion disk, as $R_{\text {in }} \propto T^{-3 / 4}$ [21]. The input seed photons have higher temperature compared to the inner disk temperature implying a smaller emission region. As a consequence they could originate from the NS surface or from the inner part of the disk such as the optically thick boundary layer[21]. The seed photons interact in a hot and optically thin plasma hypothesized to be around or inside the extended accretion disk, and eventually causing the high energy Comptonized component observed in our sample.

During the Soft state the disk extends downwards to the NS surface as showed by the high values of the inner disk temperature observed e.g. in $4 \mathrm{U}$ 1608-522 ( $R_{\text {in }}$ changes from $20 \mathrm{~km}$ during the soft state to $120 \mathrm{~km}$ during the hard state) [13]. In this case, Comptonization takes place in an optically thick cold plasma and the seed photons have a low temperature and could originate from 
the outer part of the disk. Because of the extension of the disk close to the NS, the boundary layer is optically thick and could be the region that gives rise to the Comptonization.

The Hard and Soft states observed in our sample correspond to various ratios of Luminosity to the Eddington Luminosity. In fact, estimating the bolometric luminosity of each source (see the corresponding reference for each source) the $\mathrm{L} / \mathrm{L}_{E d d}$ ranges from 0.01 (for $4 \mathrm{U} 1812-12$ ) to 0.9 (for $4 \mathrm{U} 1722-30 \mathrm{in}$ Soft state), as expected for the Atoll sources (assuming a $\mathrm{L}_{E d d}$ of $1.9 \times 10^{38} \mathrm{erg}$ $\mathrm{s}^{-1}$ for a NS of $\left.1.4 M_{\odot}\right)$. Moreover this ratio decreases from the Soft to the Hard states, implying variation in the mass accretion rates. From the Luminosity diagram we also confirm that the $\mathrm{Z}$ sources are in general more luminouses than the Atoll type implying a higher mass accretion rate.

All these informations are clues that the spectral behaviour between the NS systems and BH system are very similar, especially in the Hard state during which high temperatures of the corona are detected and the spectra extends up to $200 \mathrm{keV}$. This confirms that the "corona temperature" method is not a valid tool to discern from BH and NS systems.

\section{References}

[1] Tarana, A., Bazzano A., Ubertini P., PoS(integral08)044, 2008.

[2] Barret, D., McClintock, J. E., \& Grindlay, J. E. 1996, ApJ, 473, 963

[3] Barret, D., Olive, J. F., Boirin, L., Done, C., Skinner, G. K., \& Grindlay, J. E. 2000, ApJ, 533, 329

[4] Tavani M. \& Barret D. AIP 410, 75, 1997

[5] Zdzdiarski A. A., et al. MNRAS 301, 435, 1998

[6] Di Salvo, T., Stella, L., Robba, N. R., et al. 2000, ApJ, 544, L119

[7] Asai K., Dotani, T., Mitsuda, K. et al. 1994 PASJ, 46, 479

[8] Bird,A. J., et al., ApJS 170, 175, 2007

[9] Tarana, A., Bazzano, A., Ubertini, P. et al. 2006, ESA SP, 622

[10] Tarana, A. et al. proceeding of the 'The Extreme Universe in the Suzaku Era' Kyoto, Japan, 2006

[11] Tarana, A., Bazzano, A., Ubertini et al. 2006 A\&A 448, 335

[12] Tarana A., Bazzano B., Ubertini P. \& Zdziarski A.A., ApJ 654, 494, 2007

[13] Tarana A., Bazzano A., Ubertini P., ApJ 688, 1295, 2008.

[14] Tarana A., Belloni T., Homan J., Mendez M., Bazzano A., Ubertini P. in preparation.

[15] Tarana A., Bazzano A., Ubertini P. PoS(integral08)046, 2008.

[16] Markoff, S., Nowak, M. A., \& Wilms, J. 2005, ApJ, 635, 1203

[17] Poutanen, J., \& Coppi, P. 1998, Phys. Scr., T77, 57

[18] Titarchuk, L., \& Zannias, T. 1998, ApJ, 493, 863

[19] Migliari, S., et al. 2007, ApJ 671, 706

[20] Farinelli, R., et al. 2007, ApJ 662, 1167

[21] Frank J., King A.R., Raine D.J., Accretion power in astrophysics, Cambridge University Press, Cambridge 